\title{
Non-linear State Dependent Differential Riccati States Filter for Wastewater Treatment Process
}

\author{
Abdelhamid Iratni ${ }^{1}$, Reza Katebi ${ }^{2}$, Mohammed Mostefai ${ }^{3}$ \\ ${ }^{1}$ Centre Universitaire de Bordj Bou Arréridj, \\ El-Anasser, 34265, ALGERIA, \\ iratni@gmail.com \\ 2 Industrial Control Centre, Department of Electronic and Electrical Engineering, \\ University of Strathclyde, UK, \\ r.katebi@eee.strath.ac.uk \\ ${ }^{3}$ Laboratoire d'Automatique de Sétif, Department of Electrical Engineering, \\ University of Ferhat Abbas, \\ Sétif, ALGERIA, \\ mostefai@univ-setif.dz
}

\begin{abstract}
The most important issues relating to monitoring, quality control and prediction models for environmental protection in the treatment plant waste water are based on the amount of information and measures that are available. The key step in controlling and monitoring the plant is to obtain an accurate and robust estimate of the states model. The paper focuses on estimating non-measurable physical states of wastewater treatment system, which are unavailable because of difficulties techniques or the high cost of physical sensors. The developed filter is dealing with the non-linearity describing the system. The Activated Sludge Process (ASP) as the biological technique most commonly used wastewater treatment, attracts much attention the research community. We developed for this class of processes a robust non-linear estimator known as "state-dependent differential Riccati filter (SDDRF). The sensor software is simple to implement and has a computational cost relatively low. The results are compared with the extended Kalman filter (EKF) to demonstrate the improved performance of the filter SDDRF. The filter allows the online monitoring of process variables, which are not directly measurable. The simulation results prove the advantage of using this approach.
\end{abstract}

Keywords: wastewater system, nonlinear estimation, EKF, State dependent Riccati equation.

\section{Introduction}

The wastewater systems have usually poor and inaccurate instrumentation because of their complexity and harsh environment. Hence, very little information is available for monitoring and control. This prevents the monitoring of effluent quality and, therefore, the pollution standards cannot be guaranteed. Although some recent physical sensors provide measurements online, but for many reasons, their cost, maintenance or difficulty in placing them have limited their installation and use. Hence the design and implementation of soft sensors are still relevant and useful. The aim of this work is to propose nonlinear estimator that can be easily implemented on a real process.

Estimator developed here is applied to a simple model of ASP reported in [1]. Application to a benchmark simulator proposed by the European group COST 624 [2] will be conducted in a second phase.

Some estimation techniques have been proposed for wastewater systems in the literature. These are as follows:
- The extended Kalman filter is widely accepted and used in industry, but its convergence is difficult to prove, and the resulting estimates are highly dependent on the precision of model used [3], [4].

- The extended asymptotic observer [5] is suggested for the biological processes when the parameters of the mode are not known, but the method is not suitable for real time implementation.

- Observer-based intervals method [6] is proposed to design robust estimator but the method is based on linear models and hence not appropriate when the nonlinearities are dominant [7].

The purpose of this paper is to develop an observer sufficiently accurate to estimate the states of ASP for real time monitoring and control. The estimation algorithm is based on state dependent differential Riccati method. The proposed estimation has several useful features. It incorporates the well-known nonlinearities of the processes; it addresses the issues of high computational requirements and potentially relaxes the overly restrictive observability and controllability requirements. 
The second objective of this paper is to produce a comparative study of EKF and the SDDRF with respect to estimation accuracy, robustness and computation load. The paper is organized as follow: the modeling of the continuous wastewater treatment is briefly described in Section 2. Section 3 is dedicated to the extended Kalman filter and state dependent differential Riccati filer algorithm. The comparison between the two filters is illustrated via simulation studies in Section 4 . A general conclusion ends the paper.

\section{Bioprocess Model}

The studied wastewater system for non-linear states estimation is an Activated Sludge Process (ASP). This is the most common suspended growth process used for wastewater treatment. This biological process is an aerobic process and takes place in the aeration tank, where the wastewater is aerated with oxygen including carbonaceous oxidation and nitrification. The main difficulty is the removal of nitrogen that needs a treatment cycle, i.e. a phase of anoxia. Therefore, the process consists of two different phases: aerobic where carbon pollution is eliminated and nitrogen Ammonia is oxidized to nitrate and a phase where anoxia nitrate and nitrite nitrogen by denitrification are treated, they are converted into nitrogen gas. In practice, the phase of anoxia is upstream of the aerobic phase to ensure the necessary quantity of carbon pollution of the denitrification.

European action COST 624 proposes a simulator of a conventional ASP with its settlers in the secondary treatment of municipal two are not ventilated compartments while three are ventilated. The secondary settler is described as a series of 10 layers, dimensional model based on the Takacs model [8]. The simulation of biological process is based on the model ASM1 (Activated Sludge Model ASM1 [9]).

For the design of an estimator, the use of such models (13 states) is complicated. So we adopted a simple model, widely used in literature, but it faithfully respects the objectives of the process with an aeration tank and a secondary clarifier as displayed in Figure 1 , where $Q$ represents the secondary influent flow rate; $Q_{r}$ the return sludge flow rate; $Q_{w}$ the waste activated-sludge flow rate and $X_{e}$ the effluent suspended solids.

The mass balance around the aerator and the settler gives the following set of non-linear differential equations [10]:

$$
\begin{aligned}
\frac{d X(t)}{d t} & =\mu(t) X(t)- \\
& -D(t)(1+r) X(t)+r D(t) X_{r}(t) \\
\frac{d S(t)}{d t} & =-\frac{\mu(t)}{Y} X(t)- \\
& -D(t)(1+r) S(t)+D(t) S_{i n} \\
\frac{d C(t)}{d t} & =-\frac{K_{0} \mu(t)}{Y} X(t)-D(t)(1+r) C(t) \\
& +K_{L a}\left(C_{S}-C(t)\right)+D(t) C_{i n}
\end{aligned}
$$

$$
\begin{aligned}
\frac{d X_{r}(t)}{d t} & =D(t)(1+r) X(t)- \\
& -D(t)(\beta+r) X_{r}(t)
\end{aligned}
$$

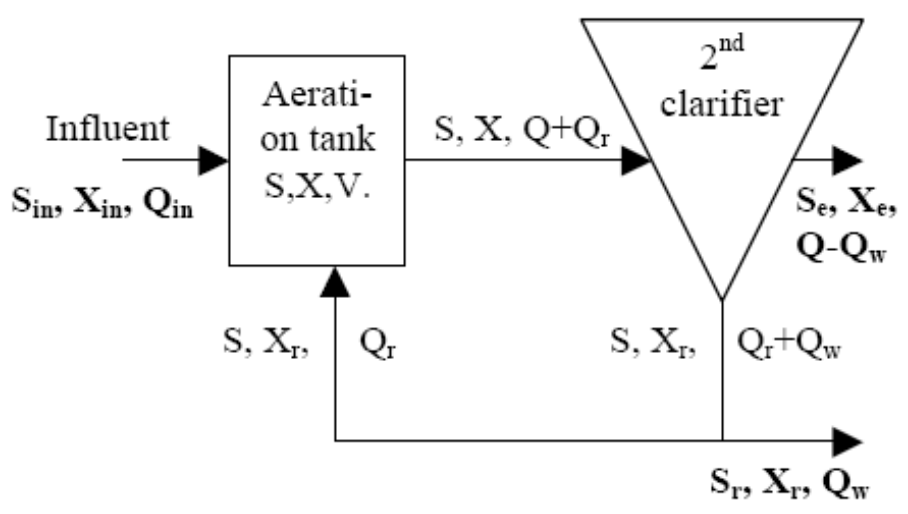

Figure 1. Activated sludge reactor used for the observers applications.

wastewater. The plant consists of a bioreactor, almost completely separated in 5 mixed compartments and a secondary settler. The first where $X(t), S(t), C(t)$ and $X_{r}(t)$ are the state variables representing the biomass, the substrate, dissolved oxygen, and the recycled 
biomass concentrations, respectively, $D(t)$ is the dilution rate, $r$ and $\beta$ represent, respectively, the ratio of recycled flow to influent flow and the ratio of waste flow to influent flow, $S_{i n}$ and $C_{i n}$ correspond to the substrate and dissolved oxygen concentrations in the feed stream, respectively. The kinetics of the cell mass production are defined in terms of the specific growth rate $\mu$ and the yield of cell mass $Y$; the term $K_{0}$ is a constant, $C_{S}$ is the maximum dissolved oxygen concentration and $K_{L a}$ represents the oxygen mass transfer coefficient.

The specific growth rate $\mu$ is a key parameter for the description of biomass growth and is known to be a complex function of many physico-chemical and biological factors like the biomass concentration, the substrate concentration, the dissolved oxygen concentration, the $\mathrm{pH}$, the temperature, and various others inhibitors. Many different analytical laws have been suggested for modelling this parameter. The most popular one is certainly the Monod law. Here we assume that the specific growth rate depends on substrate, dissolved oxygen concentrations and several kinetic parameters. The kinetic model is then given by Olsson model [11]

$$
\mu(t)=\mu_{\max } \frac{S(t)}{K_{s}+S(t)} \frac{C(t)}{K_{c}+C(t)}
$$

where $\mu_{\max }$ is the maximum specific growth rate, $K_{s}$ is the so-called affinity constant, expressing the dependency of the degradation rate on the concentration of pollutant $S$, and $K_{c}$ is the saturation constant.

\section{Estimators Design}

The nonlinear estimation problem involves constructing estimates of the state vector based on the measurement history [12]. The knowledge of process parameters is assumed to be known. The system is assumed to be piecewise observable and controllable.

Under these assumptions, the EKF [4] is presented in the next section.

\section{Extended Kalman Filter (EKF)}

The Kalman filter is an optimal stochastic linear filter that allows the estimation of the states of a system based on linear state space model. The extended Kalman filter uses local linearization to extend the scope of the Kalman filter to systems described by nonlinear functions [13].

This section presents the EKF algorithm. The state of the system at time $t$ is modelled as a stochastic variable $x$. The evolution of the state in time is expressed by:

$$
\begin{aligned}
& \dot{x}(t)=f(x(t), u(t), t)+G(t) w(t) \\
& y(t)=h(x(t), t)+v(t)
\end{aligned}
$$

Where $x(t), u(t)$ and $y(t)$ are state, input and measurement vectors. $f(x(t), u(t), t)$ and $h(x(t), t)$ are assumed to be continuously differentiable, and $w(t)$ is the $n$-dimensional process noise vector which is assumed to be a zero-mean, Gaussian process with the $n^{*} n$ covariance matrix $Q(t) . \quad v(t)$ is the $m$-dimensional measurement noise vector which is assumed to be a zero-mean, Gaussian process with variance $R(t)$. The process noise and the measurement noise are assumed to be uncorrelated.

The extended Kalman filter algorithm proceeds as follow:

Initialize: $\hat{x}\left(t_{0}\right)=\hat{x}_{0}, P_{0}=E\left\{\tilde{x}\left(t_{0}\right) \tilde{x}^{T}\left(t_{0}\right)\right\}$

Gain: $K(t)=P(t) H^{T}(\hat{x}(t), t) R^{-1}(t)$

\section{Covariance:}

$$
\begin{aligned}
& \dot{P}(t)=F(\hat{x}(t), t) P(t)+P(t) F^{T}(\hat{x}(t), t)- \\
& \quad-P(t) H^{T}(\hat{x}(t), t) R^{-1}(t) H(\hat{x}(t), t) P(t)+(10) \\
& \quad+G(t) Q(t) G^{T}(t)
\end{aligned}
$$

\section{Estimate:}

$$
\hat{\dot{x}}=f(\hat{x}(t), u(t), t)+K(t)[y(t)-h(\hat{x}(t), t)]
$$

Based on the non-linear differential equations (1) to (4), the linearized matrices for EKF are:

$$
\left.F(\hat{x}(t), t) \equiv \frac{\partial f}{\partial x}\right|_{\hat{x}(t)}=\left[\begin{array}{llll}
F_{11} & F_{12} & F_{13} & F_{14} \\
F_{21} & F_{22} & F_{23} & F_{24} \\
F_{31} & F_{32} & F_{33} & F_{34} \\
F_{41} & F_{42} & F_{43} & F_{44}
\end{array}\right]
$$

Where

$$
\begin{aligned}
& F_{11}=\mu(t)-(D(t)(1+r)), \quad F_{12}=0, \quad F_{13}=0, \\
& F_{14}=r D(t) \\
& \Delta=Y\left(K_{c}+\hat{C}(t)\right)\left(K_{s}+\hat{S}(t)\right)^{2}
\end{aligned}
$$




$$
\begin{aligned}
& F_{21}=-\frac{\mu(t)}{Y}, \\
& F_{22}=\mu_{\max } \hat{X}(t) \hat{C}(t) K_{s} D(t)(1+r) / \Delta, \\
& F_{23}=-\mu_{\max } \hat{X}(t) \hat{S}(t) K_{c} / \Delta, \\
& F_{24}=0, F_{31}=-\frac{K_{0} \mu(t)}{Y}, \\
& F_{32}=-\mu_{\max } K_{0} \hat{X}(t) \hat{C}(t) K_{s} / \Delta, \\
& F_{33}=\mu_{\max } K_{0} \hat{X}(t) \hat{S}(t) K_{c}\left((-D(t)(1+r))-K_{L a}\right) / \Delta \\
& F_{34}=0, F_{41}=D(t)(1+r), F_{42}=0, F_{43}=0, \\
& F_{44}=D(t)(\beta+r),
\end{aligned}
$$

And

$$
\left.H(\hat{x}(t), t) \equiv \frac{\partial h}{\partial x}\right|_{\hat{x}(t)}=\left[\begin{array}{llll}
0 & 1 & 0 & 0 \\
0 & 0 & 1 & 0
\end{array}\right]
$$

\section{State Dependent Differential Riccati Filter (SDDRF)}

The state-dependent Riccati equation (SDRE) method, developed over the past several years, is one a method dealing with nonlinear process dynamics, with promising performance characteristics. It has been used in nonlinear filter development and control designs for some nonlinear benchmark problems for state estimation [14], [15].

Consider the same system dynamics given by (6) and (7), and also assume that the nonlinear dynamics can be formulated into the statedependent coefficient form as follows:

$$
\begin{aligned}
& \dot{x}(t)=A(x) x(t)+B(x) u(t)+G(t) \\
& y(t)=C(x) x
\end{aligned}
$$

The estimate state $\hat{x}$ is given by

$$
\dot{\hat{x}}=A(\hat{x}) \hat{x}+K(t)[y-C(\hat{x}) \hat{x}], \hat{x}(0)=\hat{x}_{0}
$$

where the filter gain is computed as

$$
K(t)=P(t) C^{T}(\hat{x}) R^{-1}
$$

and $P(t)$ is the solution to the differential Riccati equation:

$$
\begin{aligned}
& \dot{P}(t)=P(t) A^{T}(\hat{x})+A(\hat{x}) P(t)- \\
& P(t) C^{T}(\hat{x}) R^{-1} C(\hat{x}) P(t)+Q
\end{aligned}
$$

In state space form, the matrices describing the nonlinear dynamics of equations (1) to (4) can be written as:

$$
\begin{aligned}
& A(x)=\left[\begin{array}{cc}
\mu(t)-(D(t)(1+r)) & 0 \\
-\frac{\mu(t)}{Y} & -D(t)(1+r) \\
-\frac{K_{0} \mu(t)}{Y} & 0 \\
D(t)(1+r) & 0
\end{array}\right. \\
& \left.\begin{array}{cc}
0 & r D(t) \\
0 & 0 \\
-D(t)(1+r)-K_{L a} & 0 \\
0 & D(t)(\beta+r)
\end{array}\right] \\
& B(x)=\left[\begin{array}{cc}
0 & 0 \\
D(t) & 0 \\
0 & D(t) \\
0 & 0
\end{array}\right], C(x)=\left[\begin{array}{llll}
0 & 1 & 0 & 0 \\
0 & 0 & 1 & 0
\end{array}\right] \\
& G(t)=\left[\begin{array}{c}
0 \\
0 \\
K_{l a} C_{s} \\
0
\end{array}\right]
\end{aligned}
$$

The matrices $A(x), B(x)$ and $C(x)$ in $(17,18$ and 19) are not unique, but they should be constructed such that the resulting linear system is locally observable and controllable.

\section{Simulation Studies}

In this section, two simulated scenarios will be studied to demonstrate the performance of the SDDRF in comparison with EKF to estimate the states variables of the process described by the differential equations (1) to (4). The measurements are assumed to be dissolved oxygen $C(t)$ and the substrate $S(t)$.

The following values of the model and kinetic parameters are used in the simulation: $Y=0.65$, $r=0.6, \quad \beta=0.2, \alpha=0.018 \mathrm{~m}^{-3}, K_{0}=0.5, C_{S}=10$ $\mathrm{mg} / \mathrm{l}, S_{i n}=200 \mathrm{mg} / \mathrm{l}, C_{i n}=0.5 \mathrm{mg} / \mathrm{l}, \mu_{\max }=0.15 \mathrm{~h}^{-1}$, $K_{S}=100 \mathrm{mg} / \mathrm{l}$ and $K_{c}=2 \mathrm{~m} / \mathrm{l}$. The initial condition and initial values for SDDRF and EKF: $X(0)=215 \mathrm{mg} / \mathrm{l}, C(0)=6 \mathrm{mg} / \mathrm{l}, S(0)$ $=55 \mathrm{mg} / \mathrm{l}, X_{r}(0)=400 \mathrm{mg} / \mathrm{l}$.

The process noise is assumed to be $w \sim N(0$, $0.05)$ and the sensor noise is $v \sim N(0,0.1)$.

Scenario 1: It is assumed that Dilution rate $D(t)$ and air flow rate $W(t)$ are constant in time, then the outputs from the model with the estimates from SDDRF and the EKF are compared. 
Based on the EKF and SDDRF design procedures described in the previous section, Simulation results are shown in Figure 2a and Figure 2b. It illustrates the evolution of the output states and their corresponding real nonlinear variables. Both SDDRF and EKF can estimate the state-variables well. However, it is found that the standard deviation of the estimation errors for SDDRF is better than EKF as shown in Table 1.
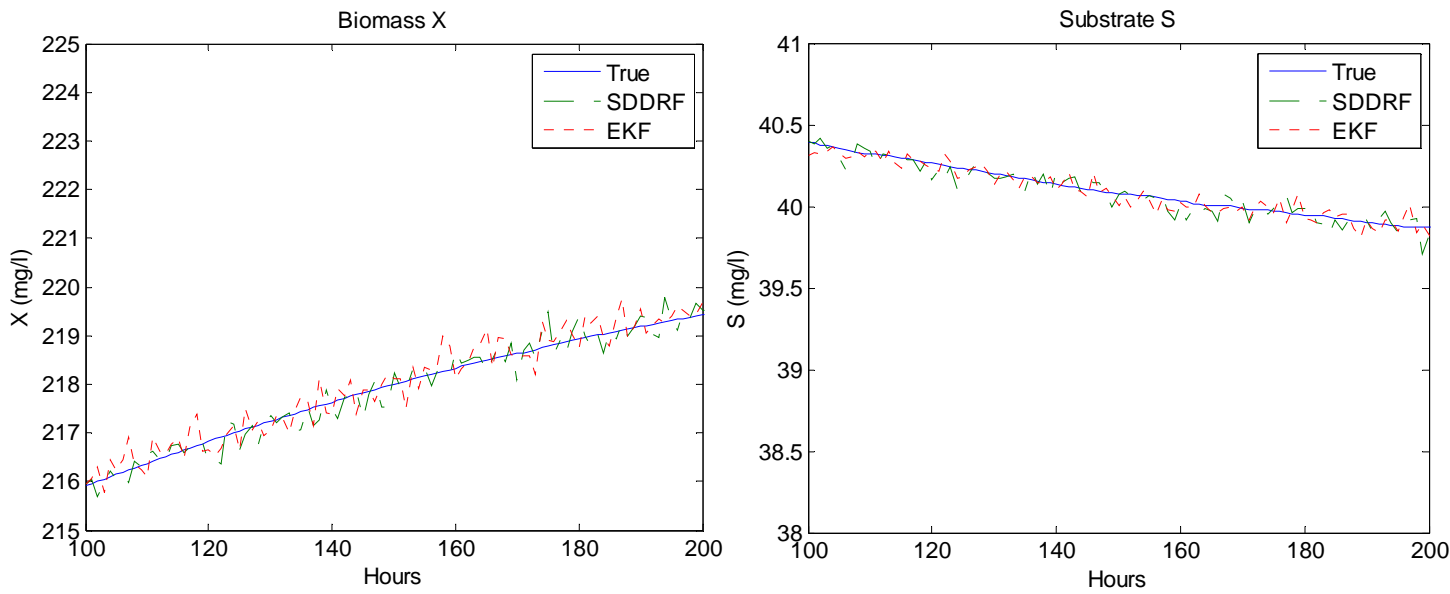

Figure 2a. Comparison of true states $X(t)$ and $S(t)$ with their estimates.
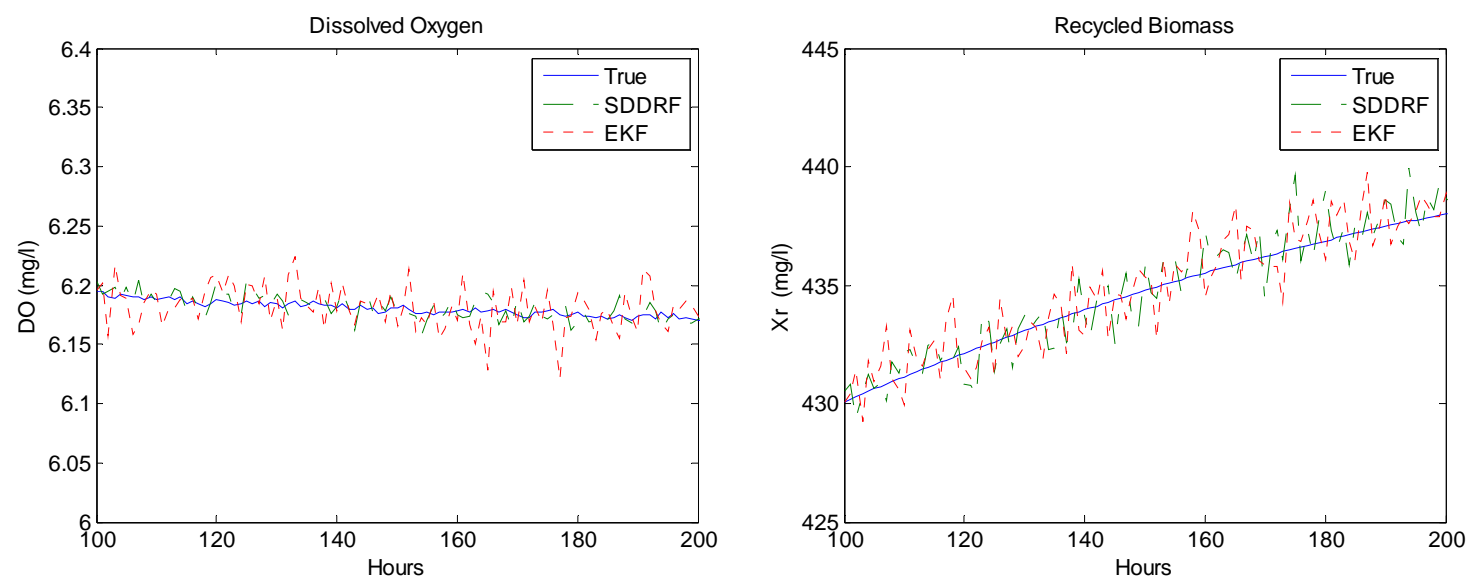

Figure 2b. Comparison of true states $C(t)$ and $X_{r}(t)$ with their estimates.

Table 1. Standard deviations of SDDRF and EKF for the state variables.

\begin{tabular}{ccccc}
\hline & $\mathbf{X}(\mathbf{t})$ & $\mathbf{S}(\mathbf{t})$ & $\mathbf{C}(\mathbf{t})$ & $\mathbf{X}_{\mathbf{r}}(\mathbf{t})$ \\
\hline SDDRF & 0.21761 & 0.049041 & 0.0030626 & 0.92035 \\
\hline EKF & 0.53554 & 0.062001 & 0.0047854 & 2.1329 \\
\hline
\end{tabular}


Scenario 2: The Dilution rate $D(t)$ and the airflow rate $W(t)$ are assumed to have several step changes in time and magnitude. Results are presented in Figure 3a and $3 b$ for the four variables. It can be observed that the SDDRF provide better performances compared to EKF. However, it is found that the convergence rate is highly related to the choice of initial values. Since this is a nonlinear estimation problem where the convergence can only be local, good initial guess may be beneficial for the fast convergence. The standard deviation of the estimation errors for the two filters is given in Table 2.
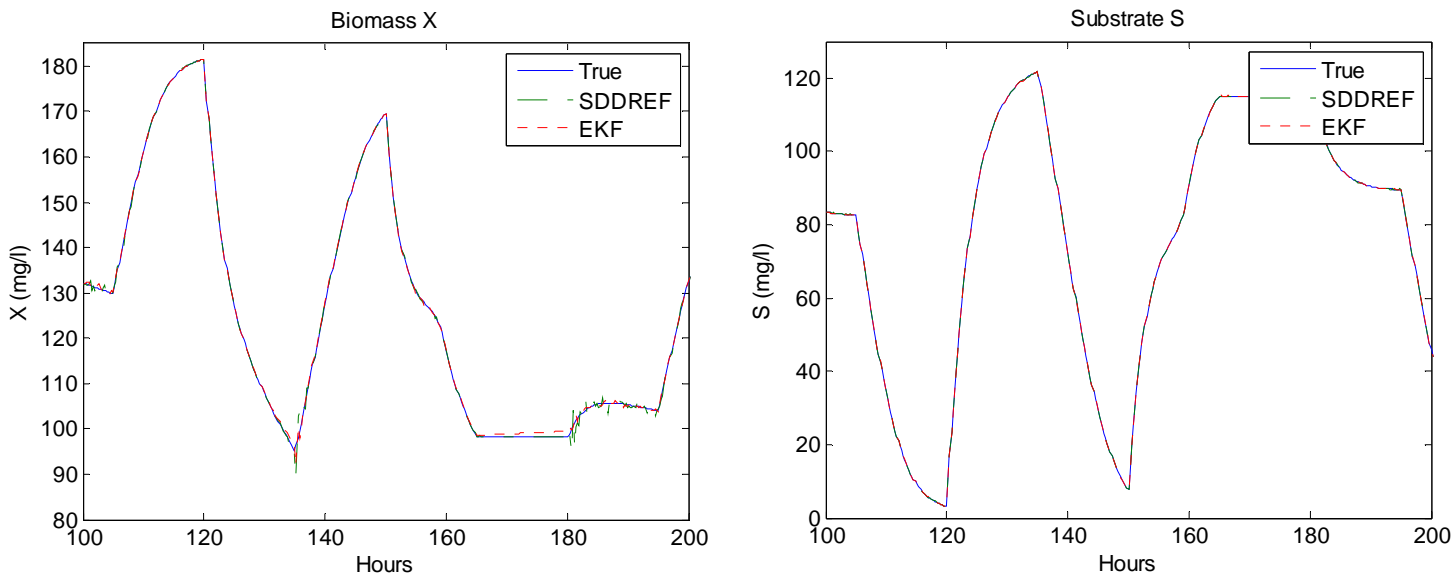

Figure 3a. Comparison of true states $X(t)$ and $S(t)$ with their estimates.
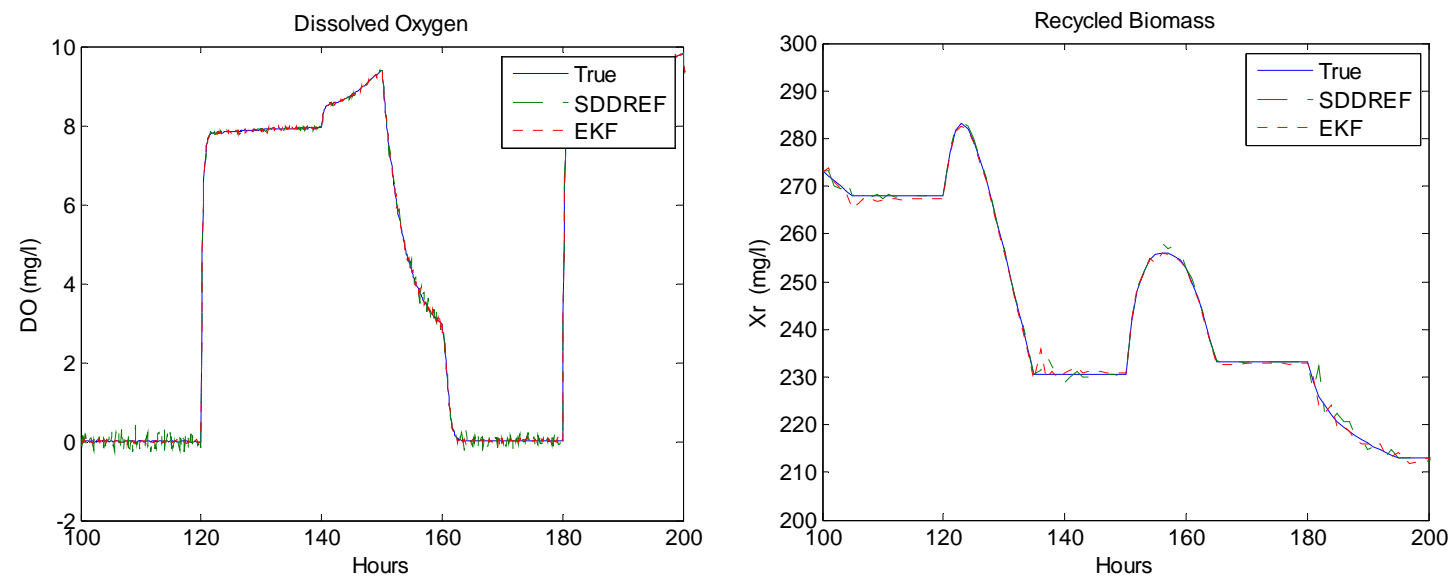

Figure 3b. Comparison of true states $C(t)$ and $X_{r}(t)$ with their estimates.

Table 2. Standard deviations of SDDRF and EKF for the state variables.

\begin{tabular}{ccccc}
\hline & $\mathbf{X}(\mathbf{t})$ & $\mathbf{S}(\mathbf{t})$ & $\mathbf{C}(\mathbf{t})$ & $\mathbf{X r ( t )}$ \\
\hline SDDREF & 0.47627 & 0.05808 & 0.028733 & 2.0944 \\
\hline EKF & 1.8087 & 0.053968 & 0.0153 & 6.5501 \\
\hline
\end{tabular}


The robustness of the above observers in the presence of modelling, sensor and computational errors were also investigated. Increasing the process and measurement variances $\mathrm{Q}$ and $\mathrm{R}$ does this. This is an important study since in reality; the proposed schemes have to work in noisy environments. The squared estimation error (SQR) is defined as

$$
S Q R=\int_{0}^{T} \sqrt{(X-\hat{X})^{2}+(S-\hat{S})^{2}+(C-\hat{C})^{2}+\left(X_{r}-\hat{X}_{r}\right)^{2}} d t
$$

For various process and measurement noise, the results are shown in Tables 3 and 4 . It is clear that the SDDRF has better performance as noise increased.

Table 3. Squared error performances of estimators as function of process noise variance.

\begin{tabular}{lll}
\hline $\mathbf{Q}(\mathbf{t})$ & SDDRF & EKF \\
\hline 0.05 & 5.1613 & 4.8225 \\
\hline 0.1 & 7.4689 & 17.2984 \\
\hline 0.5 & 16.6510 & 245.5748 \\
\hline 1 & 32.8298 & 499.3076 \\
\hline
\end{tabular}

Table 4. Squared error performances of estimators as function of measurement noise variance.

\begin{tabular}{lll}
\hline $\mathbf{R}(\mathbf{t})$ & SDDRF & EKF \\
\hline 0.1 & 5.4953 & 6.6443 \\
\hline 0.5 & 9.1259 & 11.7735 \\
\hline 1 & 11.9203 & 20.0615 \\
\hline 10 & 73.7655 & 260.4276 \\
\hline
\end{tabular}

\section{Conclusion}

In this work, a new soft sensor for estimating the unmeasured state variables in wastewater system was presented. A comparative study between SDDRF and EKF filters was presented. The proposed filter has proved to have better estimation accuracy. The filer can be a costeffective alternative to physical sensors for biomass and recycled biomass estimation.

\section{Acknowledgements}

The first author is grateful to the Ministry of Higher Education and Scientific Research in Algeria for granting permission to carry out this research. Financial support from the Algerian Government is gratefully acknowledged.

\section{REFERENCES}

1. NEJJARI, F., B. DAHHOU, A. BENHAMMOU, G. ROUX, Non-linear Multivariable Adaptive Control of an Activated Sludge Wastewater Treatment Process, International Journal of Adaptive Control and Signal Processing, 13, 1999, pp. 347-365.

2. COPP, J. B., The COST Simulation Benchmark: Description and Simulator Manual, (COST Action 624 \& COST Action 682), Office for Official Publications of the European Communities, Luxembourg, 2002.

3. KALMAN, R. E., R. S. BUCY, New Results in Linear Filtering and Prediction Theory, Journal of Basic Engineering, 1961, pp. 95-108.

4. BENAZZI, F., R. KATEBI, J. WILKIE, Application of Extended Kalman Filter to Activated Sludge Process, EU Research Training Network (HPRN-CT-200100200), internal report, 2003.

5. BASTIN, G., D. DOCHAIN, On-line Estimation and Adaptive Control of Bioreactors, Elsevier Publisher, 1990.

6. HADJ-SADOK, M. Z., J. L. GOUZÉ, Estimation of Uncertain Models of Activated Sludge Processes with Interval Observers, Journal of Process Control 11. Elsevier Publisher, 2001.

7. GOMEZ-QUINTERO, C. S., I. QUEINNEC, Robust Estimation for an Uncertain Linear Model of an Activated Sludge Process, 2002 IEEE International Conference on Control Applications, 2002, pp. 972-977.

8. TAKACS, I., G. G. PATRY, D. NOLASCO, A Dynamic Model of the Clarification Thickening Process, Water Research, 25(10), 1991, pp. 1263-1271. 
9. HENZE, M., JR. GREDY, W. GUJER, G. MARAIS, T. MATSUO, Activated Sludge Model $\mathbf{n}^{\circ} \mathbf{1}$, IAWQ Scientific and Technical report $\mathrm{n}^{\circ} 1$. London, 1986.

10. KATEBI, R., M. JOHNSON, J. WILKIE, Control and Instrumentation for Wastewater Treatment Plants, Springer edition, 1999.

11. G. OLSSON, State of the Art in Sewage Treatment Plant Control, American Institute of Chemical Engineers, Symposium Series, 1976, pp. 52-76.

12. DUZINKIEWICZ, K., A. BOROWA, K. MAZUR, M. GROCHOWSKI, M. A. BRDYS, K. JEZIOR, Leakage Detection and Localisation in Drinking Water Distribution Networks by MultiRegional PCA, Studies in Informatics and Control, Vol. 17(2), 2008, pp. 135-152.
13. MAYBECK, P. S., Stochastic Models, Estimation and Control. New York: Academic Press, 1982.

14. AZEMI, A., E. E. YAZ, Comparative Study of Several Nonlinear Stochastic Estimators, Proceedings of the 38th IEEE Conference on Decision and Control, Phoenix, AZ, vol. 5, 1999, pp. 4549-4554.

15. MRACEK, C. P., J. R. CLOUTIER, C. A. D'SOUZA, New Technique for Nonlinear Estimation, Proceedings of the IEEE Conference on Control Applications, 1996, pp. 338-342. 